Revue trimestrielle sur l'image géographique et les formes du territoire

$129 \mid 2020$

Varia

\title{
La Gouvernance portuaire, un enjeu de développement en Baltique orientale
}

Portuary governance, a developmental issue in the Eastern Baltic

Gobernanza portuaria : un reto para el desarrollo del Báltico oriental

\section{Arnaud Serry et Lilian Loubet}

\section{(2) OpenEdition}

Journals

Édition électronique

URL : http://journals.openedition.org/mappemonde/4828

DOI : $10.4000 /$ mappemonde.4828

ISSN : 1769-7298

Éditeur

UMR ESPACE

Référence électronique

Arnaud Serry et Lilian Loubet, « La Gouvernance portuaire, un enjeu de développement en Baltique orientale ", Mappemonde [En ligne], 129 | 2020, mis en ligne le 10 novembre 2020, consulté le 26 novembre 2020. URL : http://journals.openedition.org/mappemonde/4828 ; DOI : https://doi.org/ 10.4000/mappemonde.4828

Ce document a été généré automatiquement le 26 novembre 2020.

\section{cc) (†) (2)}

La revue Mappemonde est mise à disposition selon les termes de la Licence Creative Commons Attribution - Pas d'Utilisation Commerciale - Partage dans les Mêmes Conditions 4.0 International. 


\title{
La Gouvernance portuaire, un enjeu de développement en Baltique orientale
}

\author{
Portuary governance, a developmental issue in the Eastern Baltic \\ Gobernanza portuaria : un reto para el desarrollo del Báltico oriental
}

Arnaud Serry et Lilian Loubet

\section{Introduction}

1 La façade maritime orientale de la mer Baltique est constituée de ports ayant pour vocation principale d'exporter des matières premières en provenance de la Russie et de la CEI (Communauté des États Indépendants) et d'approvisionner ces dernières en produits manufacturés. Les ports russes (Saint-Pétersbourg, Oust-Louga, Primorsk et Kaliningrad), baltes (Tallinn, Riga, Ventspils et Klaipeda), voire finnois (Hamina-Kotka) interagissent dans un système de desserte d'un vaste arrière-pays englobant une partie de l'Europe centrale et le monde russe. Ici, les ports forment des nœuds de transport primordiaux, en particulier dans un trafic de transit où la complémentarité a fait place peu à peu à une concurrence intense.

Dans ce contexte, les évolutions en matière de gouvernance portuaire constituent un enjeu majeur de développement territorial. L'espace baltique, région en profonde mutation depuis plus de 25 ans, apparait particulièrement concerné par le phénomène. L'objectif de ce papier n'est pas de produire une énième analyse des modèles ou des modalités de cette gouvernance, mais de considérer celle-ci comme un ressort de développement. L'analyse est menée à partir d'une vingtaine d'entretiens semidirectifs conduits en Baltique orientale, dans les ports de Tallinn, Hamina-Kotka et Klaipeda, réalisés auprès de différents types d'acteurs (élus, représentants des autorités portuaires, responsables de chambres de commerce, universitaires, professionnels 
portuaires, etc.) ${ }^{1}$. L'objectif de ces entretiens est d'analyser les relations ville-port ainsi que d'étudier les nouveaux jeux d'acteurs qui en découlent.

3 La première partie de cet article présente les caractéristiques et évolutions récentes de la gouvernance portuaire de la façade maritime orientale de la Baltique. La seconde distingue différents modèles régionaux de gouvernance en identifiant le rôle respectif des différents acteurs (portuaires, municipaux, régionaux, privés...). Enfin, l'impact de leurs jeux sur le développement portuaire et territorial sera étudié dans une dernière partie.

\section{Les ports de Baltique orientale : de la centralisation monopolistique à la concurrence}

\section{Évolutions contemporaines des modalités de gouvernance portuaire}

4 Traditionnellement, les rapports entre port et territoires reposent sur la relation villeport. En effet, ces liens sont historiques même si cette corrélation s'est affaiblie au cours des dernières décennies, notamment parce que les mutations du commerce international ont astreint les ports à se transformer et, souvent, à s'éloigner des villes. Désormais, le champ d'action s'est amplement étendu, intégrant une vision d'ensemble de place portuaire qui permet d'élargir le propos aux institutions locales, à la région, aux chambres de commerce et aux organisations professionnelles, se rapprochant ainsi du modèle de gouvernance hanséatique (Lévêque, 2014). Par conséquent, les autorités portuaires sont fortement incitées à réfléchir sur la pertinence à moyen et long terme de leurs stratégies et leurs politiques de développement. L'important n'est plus la tonne manutentionnée, mais les retombées économiques sur le territoire, notamment en termes de création d'emplois (Lemaire, 2012). Les ports sont des systèmes complexes et leur efficience est déterminée non seulement par leur localisation, mais aussi par les intérêts des membres de la communauté portuaire et leurs interactions sur des échelles territoriales multiples (Mickiene, 2017).

5 La gouvernance portuaire en Europe varie selon les États, avec des modes de gestion qui tendent vers un renforcement progressif de la place du privé. Cette gouvernance diffère selon le modèle portuaire adopté (figure 1), en fonction desquels les rôles du public et du privé évoluent :

$6 \quad$ Le service port (port de service public), qui repose sur un centralisme politique où l'État contrôle sa stratégie portuaire est un port totalement public sauf au sein des services de remorquage/lamanage ou de dragage/pilotage qui peuvent être délégués en partie à des entreprises privées. C'est dans des ports principalement latins que l'on observe ce modèle portuaire (Tourret, 2014).

7 Le tool port (port-outil) est un port dans lequel l'autorité portuaire relève du public (à l'image du service port), mais dont la manutention est exercée par des sociétés privées. Ce modèle permet à l'autorité portuaire de bénéficier d'une gestion public-privé tout en réduisant les risques encourus par des opérations d'investissements financiers (Burns, 2014).

8 Le landlord port (port-foncier) est un modèle portuaire qui tend à s'imposer dans la majorité des grands ports européens (Verhoeven, 2010 ; Serry et Loubet, 2019) avec un partenariat public-privé plus important puisque les superstructures, mais aussi parfois 
les infrastructures sont déléguées à des opérateurs privés dans une logique de « terminalisation » des ports (Slack, 2007).

9 Le private service port (port privé) est un port majoritairement voire exclusivement privé avec la privatisation de son administration portuaire. Cette ouverture du capital de l'autorité portuaire a comme conséquence l'apparition de sociétés portuaires privées. Ce modèle tend à se développer dans certains pays européens comme en Angleterre où les ports ne reçoivent plus de dotation publique.

Figure 1. Rôle du public et du privé dans le management portuaire

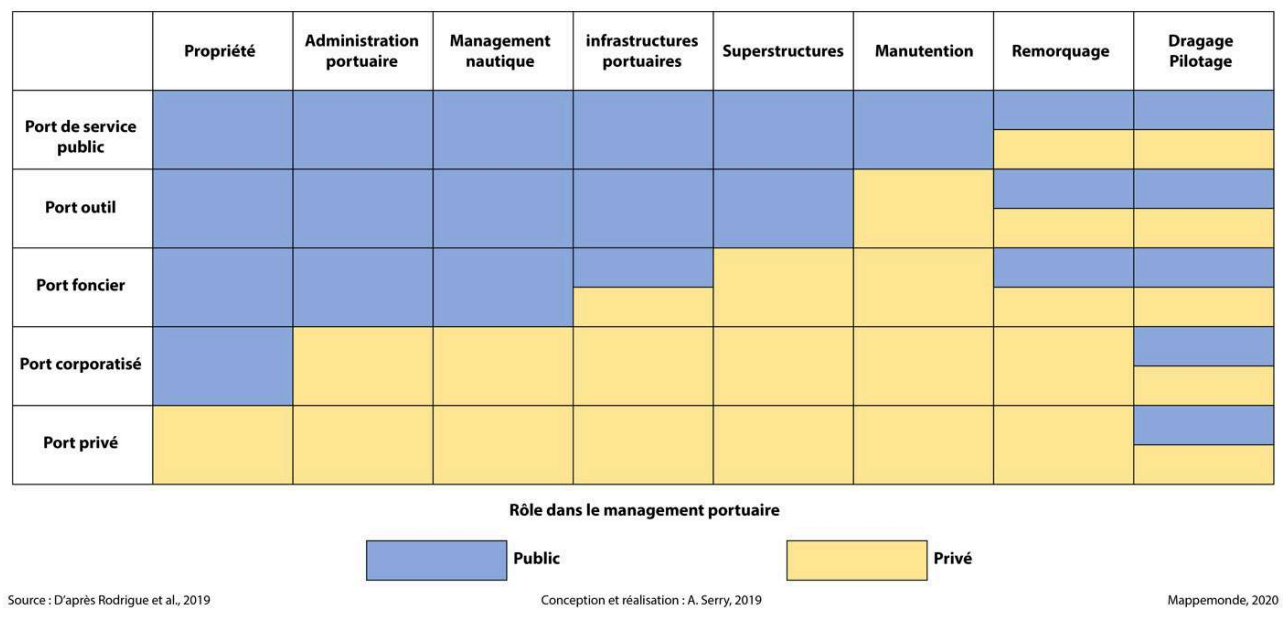

Source : A. Serry d'après Rodrigue et al., 2019

10 Les modalités de gouvernance portuaire sont marquées par une normalisation autour de l'archétype du port foncier (landlord port) (Verhoeven, 2010). Ce modèle repose sur la cession de l'exploitation portuaire à des opérateurs privés dans une logique de «terminalisation» des ports (Slack, 2007). Dans cette terminalisation, les ports sont divisés en autant de sous-ports (terminaux) qu'il y a d'opérateurs privés (Charlier et Lavaud-Letilleul, 2011). En effet, alors que le secteur public garde la gestion du patrimoine foncier, l'outillage et la main-d'œuvre sont désormais légués à des concessionnaires privés (figure 1). De ce fait, le développement du modèle de landlord port avec comme corollaire un processus de globalisation des activités manutentionnaires pose comme enjeu la gestion et la maitrise stratégique du développement urbano-portuaire.

11 Toutefois, ce schéma distingue le modèle latin dans lequel le port est sous l'influence de l'État et la configuration hanséatique qui évoque une gouvernance de proximité et qui serait un juste milieu entre le port privé et une gestion trop étatisée (Tourret, 2014). Il est caractérisé par une forte autonomie par rapport à l'état central et s'inscrit dans la longue durée. Quel que soit le modèle choisi, chaque port est soumis de manière variable aux instances politiques des différentes échelles institutionnelles. Les modalités d'acheminement du fret maritime relèvent quant à elles d'une prise de décision du secteur privé. 

juridiques et les modes de gouvernance d'une autorité portuaire (Brooks, Pallis, 2012) :

- La commercialisation est le passage d'un fonctionnement d'administration du port à une culture plus commerciale avec transfert vers le secteur privé d'activités commerciales préalablement exercées par l'autorité portuaire.

- La corporatisation est l'acquisition d'une autonomie financière par l'autorité portuaire (celle-ci restant détenue par des acteurs publics) et l'application de règles de gestion d'entreprise (Verhoeven, 2010). Les ports corporatisés sont des ports grandement privatisés, mais dans lesquels la propriété demeure publique et souvent assumée en tant qu'actionnaire majoritaire. L'autorité portuaire se comporte essentiellement comme une entreprise privée. Ce modèle de gestion est unique, car il est le seul où la propriété et le contrôle sont séparés (Rodrigue et al., 2017).

- La privatisation est l'ouverture du capital de l'autorité portuaire. La privatisation conduit en général à retirer au gestionnaire du port les attributions régaliennes de l'autorité portuaire.

Ainsi animés par des acteurs aux intérêts souvent discordants, les espaces portuaires sont propices aux confrontations de projets et conflits d'usages, notamment entre les fonctions urbaines et récréatives et les fonctions portuaires et industrielles. Par exemple, à Tallinn, l'omniprésence des paquebots et des ferries à proximité immédiate de la ville et les nuisances qui en découlent (sonores, émissions de gaz, congestion) sont à l'origine de la grogne d'une partie de la population et d'adaptations portuaires comme des droits de quai réduits pour les navires propulsés au GNL. Ces jeux d'acteurs sont à géométrie variable : rapport public-privé, interférences institutionnelles, effet de concurrence, divergence de point de vue entre gens de mer et gens de terre (Foulquier, 2009). Aussi, « le rapport à l'autorité publique demeure ambivalent, entre le besoin d'un encadrement stratégique pour voir loin et des revendications d'autonomie pour agir plus vite » (Guillaume, 2014). Cette variété d'échelles et cette multiplication du nombre de "parties prenantes» constituent le cadre général structurant les différents modes de gouvernance des systèmes portuaires mondiaux (Comtois, 2014). Ces mutations s'inscrivent dans un contexte marqué par le renforcement de la place du secteur privé dans le secteur portuaire et par l'évolution des missions et des statuts juridiques des autorités portuaires afin de les rapprocher de ceux d'entreprises privées tout en confortant leurs fonctions régaliennes et d'aménagement (Guerlet, 2013). Le mode de gouvernance territorial diffère donc selon les pays et structure les relations entre le port et la ville et plus généralement entre le port et le territoire (dans un rapport public-privé). Mais qu'en est-il en fonction de la taille de la ville portuaire? Nous prenons le cas de la ville portuaire "moyenne" où l'analyse textuelle des relations ville-port permettra de proposer une caractérisation fonctionnelle.

\section{La fin de l'organisation soviétique et les affirmations nationales}

Hérités de l'organisation du transport maritime de l'URSS, les ports de Baltique orientale restent marqués par cette dernière. La centralisation y était de mise. L'administration et la gestion de l'activité maritime et portuaire étaient placées sous la compétence du ministère de la flotte maritime au sein d'un vaste monopole intégré verticalement.

Des compagnies maritimes régionales étaient responsables de la flotte et des ports de leur territoire. La structure du commerce maritime y était liée à une compagnie de 
navigation comme la Latvian Shipping Company rattachée aux ports lettons. Les ports étaient spécialisés en fonction de leur situation sur le territoire soviétique et du rôle que leur assignait le ministère de la Marine. Ainsi, Ventspils et Klaipeda géraient à eux seuls $26 \%$ des exportations pétrolières de l'union.

Par ailleurs, la façade baltique assurait $22 \%$ du commerce maritime de l'URSS, dont les trois quarts par les ports baltes et seulement un quart par les ports russes. À la suite du démantèlement de l'URSS, il est évident que la Russie a connu une importante perte de capacité portuaire à laquelle elle a dû remédier. Ce rétrécissement de son ouverture européenne sur les mers conditionnera la politique portuaire russe jusqu'à aujourd'hui.

Dans un premier temps, à la recherche de nouveaux statuts, les ports sont restés à michemin entre collectivisme et terminalisation. Cette intégration port-armement semblait propice au développement d'une politique maritime propre à chaque état récemment indépendant. L'extrême fin du $\mathrm{XX}^{\mathrm{e}}$ siècle a été synonyme d'une mutation de l'organisation portuaire. Les républiques baltes, les régions, les villes, tous les niveaux d'autorité territoriale ont voulu contrôler les ports. Les entreprises privées ont fait leur apparition dans le secteur, d'où la réduction du rôle des compagnies maritimes à celui d'armateur.

\section{Renouveau, croissance partagée et compétitions}

Depuis le milieu des années 1990, une croissance continue du trafic et la modernisation portuaire caractérisent les ports de la Baltique orientale. Dans un premier temps, cette tendance a été partagée par la majorité des ports, aussi bien baltes que russes. Le trafic global en mer Baltique a ainsi doublé de 1997 à 2017 (passant de 420 millions de tonnes à plus de $800 \mathrm{Mt}$ ). Surtout, c'est sur la rive orientale que la croissance est la plus soutenue (figure 2).

Figure 2. Ports de plus de 10 millions de tonnes de trafic en 2017

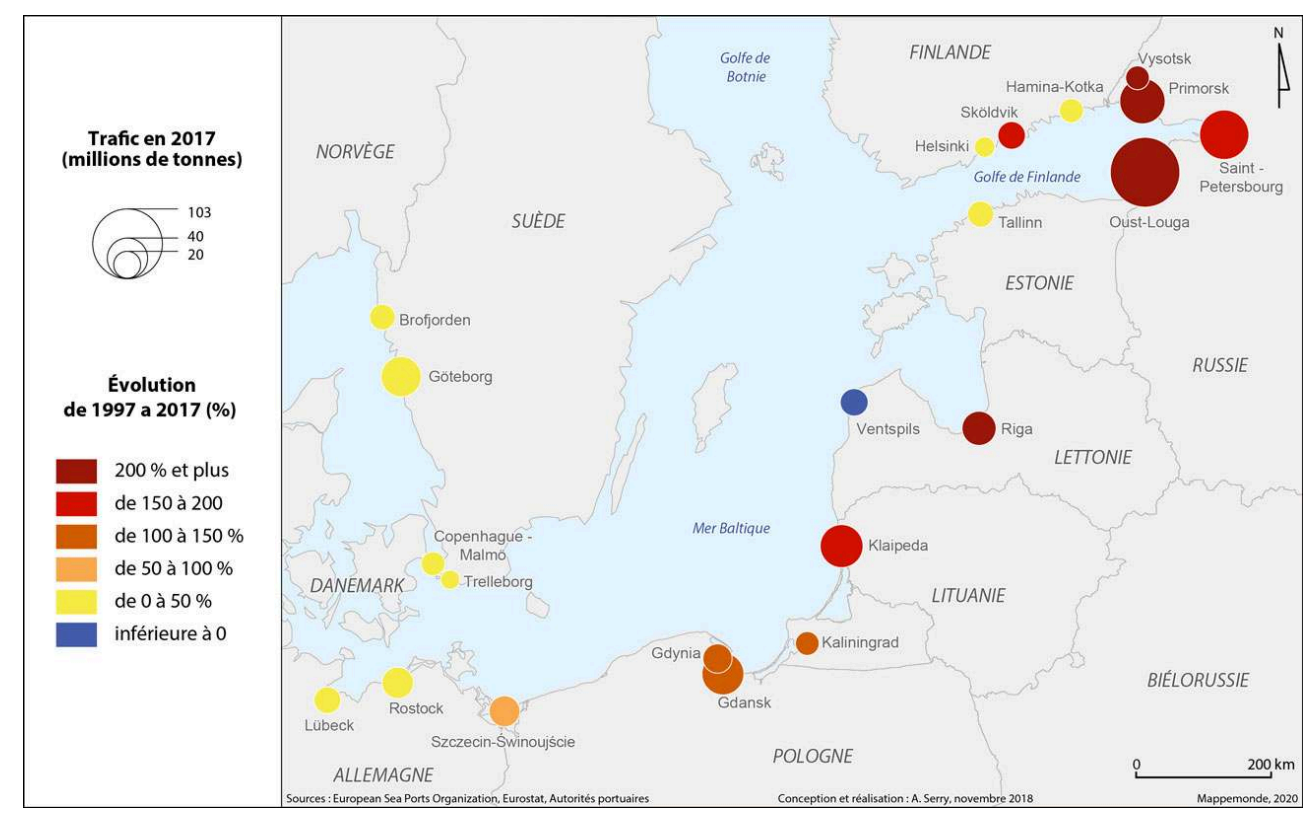

Réalisation : A. Serry 
Celle-ci repose sur trois moteurs fondamentaux : la croissance économique mondiale, les intenses mutations géopolitiques de la région et les besoins en capacité portuaire de la Russie. Les chiffres confirment le rôle majeur du golfe de Finlande dans le trafic conteneurisé, véritable Gateway de la Russie (Serry, 2018).

Les hydrocarbures occupent, devant le vrac sec, une place prépondérante dans les flux à l'exportation (Est-Ouest), dans les ports étudiés. Ils représentent, tout particulièrement le pétrole brut et le gaz naturel, un enjeu particulier en raison des fortes relations d'interdépendance existant entre l'Union européenne et la Russie dans ce domaine. Ainsi, le premier terminal GNL opérationnel depuis fin 2014 à Klaipèda, ayant pour objectif de diminuer la dépendance énergétique à l'égard de la Russie, a symboliquement été baptisé Independance.

21 Le déplacement du centre de gravité de l'espace baltique vers l'est semble une évidence au regard des réseaux maritimes. Les ports de la Baltique orientale comme Klaipeda et Riga apparaissent (avec Gdansk) comme les pôles majeurs du réseau conteneurisé dominé par Saint-Pétersbourg (figure 3).

Figure 3. Les dessertes conteneurisées en mer Baltique en 2017

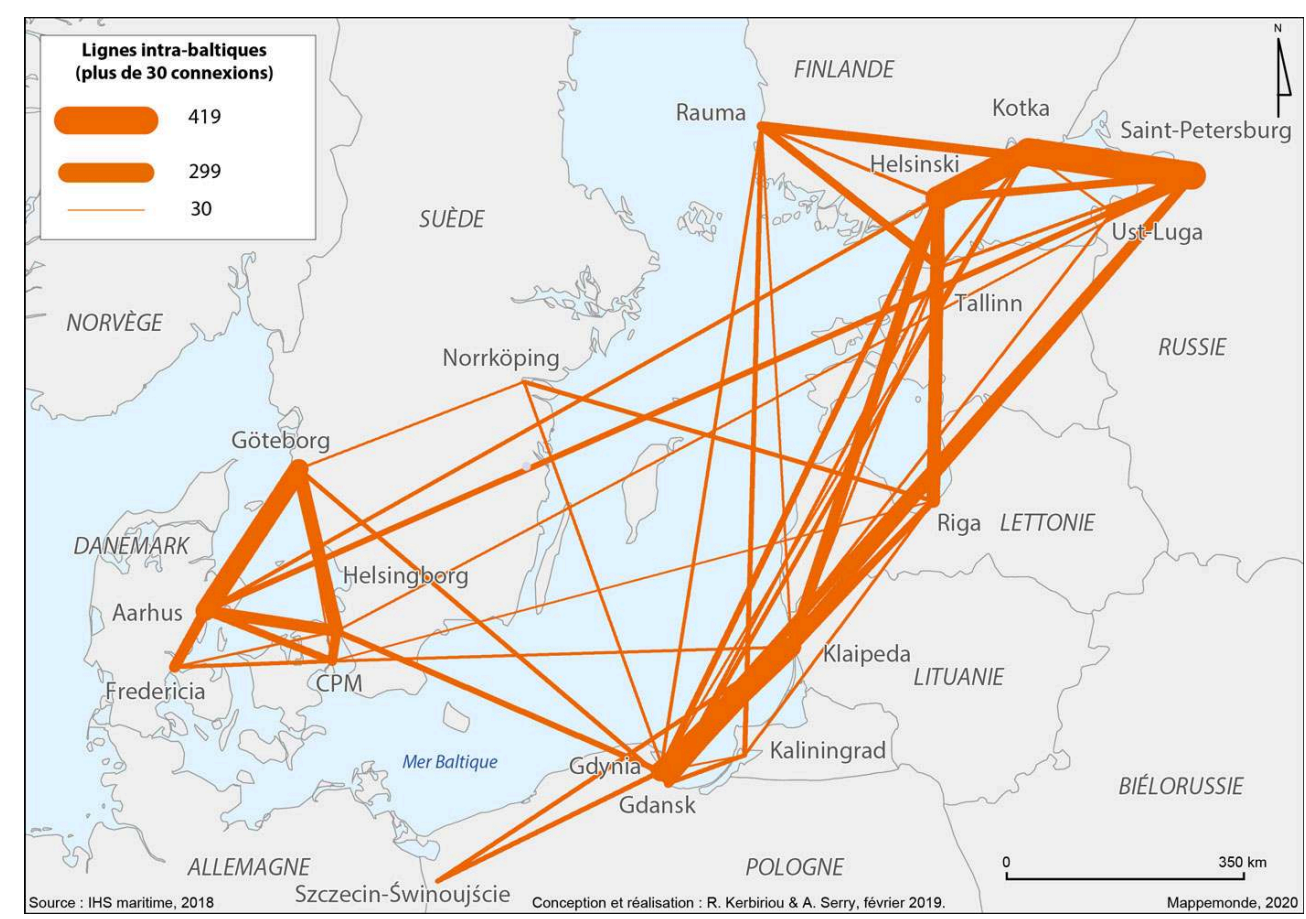

Réalisation : R. Kerbiriou et A. Serry

22 Au sein du réseau conteneurisé, le golfe de Finlande et plus largement la Baltique orientale représentent deux zones particulièrement actives. Nous observons clairement une distinction des activités maritimes et portuaires sur la rive orientale de la mer Baltique, où la mise en réseau des ports est évidente. Évidente également est la hiérarchisation comme l'illustre l'affirmation comme pivot régional du port de Gdansk. Celui-ci affiche d'ores et déjà des ambitions de leadership régional.

Les ports russes, baltes voire finnois, se distinguent et interagissent dans un système de desserte d'un vaste arrière-pays englobant premièrement le monde russe ainsi qu'une partie de l'Europe centrale. Les marchandises sont diffusées à partir de cette région 
gateway dans un milieu différent et avec des modes différents. Cet essor est lié à la mise en service de nouvelles capacités portuaires, de Gdansk à Oust-Louga (Serry, 2019). En conséquence, nous assistons à une forme d'intensification concurrentielle avec des multichoix possibles de ports maritimes. Les ports aux arrière-pays les moins diversifiés se trouvent dans des situations délicates, comme Tallinn aujourd'hui. D'autant plus que cette façade maritime est dorénavant soumise à des conditions économiques moins favorables. Le système de transport maritime régional a ainsi glissé d'une complémentarité imposée par les héritages historiques à une concurrence exacerbée et une différentiation dans le rôle des ports.

\section{Une gouvernance portuaire protéiforme en Baltique orientale}

La Baltique orientale est dominée par des ports fonciers d'état ou des ports municipaux (Mickiene, 2017). Les autorités portuaires partagent avec les gouvernements nationaux et locaux la responsabilité du développement des infrastructures (Bolevics, 2017). Malgré le poids des technostructures, de réels changements y ont été opérés. Les entreprises privées occupent une place de plus en plus importante même si certaines organisations prennent encore parfois la forme d'entreprise publique à vocation commerciale. Les privatisations d'anciennes compagnies de manutention portuaire et l'apparition de nouvelles sociétés illustrent ce renforcement de la sphère privée désormais au cœur des activités portuaires.

Toutefois, les structures de gestion et de propriété des ports varient considérablement d'un État à l'autre, allant d'une gestion intégralement étatique à une possession totalement municipale à Kotka, en passant par une administration conjointe entre municipalité et échelon national en Lettonie.

\section{Les États baltes} échelons municipaux et nationaux (figure 4) : les autorités portuaires agissent en tant qu'institutions à but non lucratif établies dans les municipalités concernées sous le contrôle du ministère des Transports (Bolevics, 2017). Les administrations portuaires sont dominées par un conseil de 8 membres nommés, 4 représentants des autorités locales et 4 des ministères des Transports, de l'Économie, des Finances et de l'Environnement.

Le système de gouvernance dans les deux ports maritimes lettons ne suit ni la voie de la corporatisation ni celle de la commercialisation. Ils sont soumis à l'influence directe des hommes politiques municipaux et nationaux. Cette coopération des différents échelons est perçue comme un avantage plus qu'un obstacle (Bolevics, 2017). Toutefois, la faible représentation des intérêts privés dans les autorités portuaires lettones apparait comme un frein à leur développement.

«Nous croyons que, les entreprises travaillant au port (de Ventspils) sont des otages sans voix... Cela se traduit par un environnement des affaires plus pauvre, 
des dépenses inutiles et des investissements inefficaces. Pour cette raison, il serait logique de modifier le modèle de gouvernance des ports et d'inclure dans leur direction des représentants des entreprises portuaires. " Ivars Landmanis, membre du comité directeur de la Baltic Association -Transit and Logistics (30 novembre 2018, Baltic News Network : https://bnn-news.com/batl-ventspils-freeport-s-decision-toraise-fees-will-not-bring-in-more-cargoes-194517).

Figure 4. Statuts et tutelles des ports de la Baltique orientale

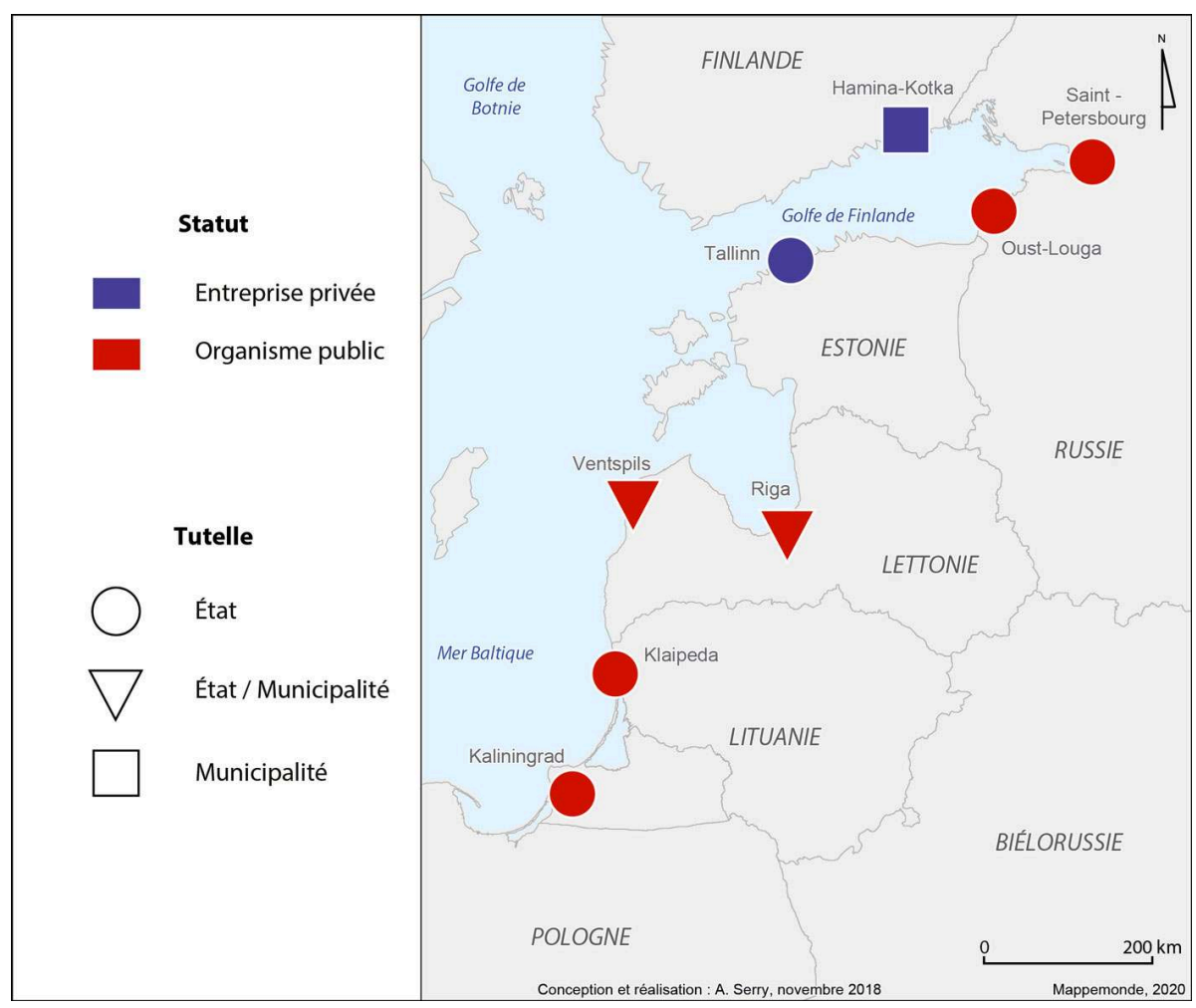

Réalisation : A. Serry

En Estonie, le port de Tallinn, dont la libéralisation a été initiée dès 1992 (Kukrus et Poldroos, 2003) est exploité par une société anonyme, dont l'État a été l'actionnaire unique jusqu'en 2018. Il a alors été privatisé et l'entreprise cotée en bourse (figure 4). Les actionnaires sont désormais l'État estonien à hauteur de $67 \%$ et des fonds d'investissement ou des investisseurs privés.

Le port de Tallinn a donc glissé vers une corporatisation et se distingue des autres ports (figure 1) notamment parce qu'il est géré en incorporant des critères de saine gouvernance d'entreprise et parce qu'il a étendu sa sphère d'influence à la chaine logistique.

31 À la tête de la compagnie se trouvent un conseil d'administration qui s'occupe de la gestion quotidienne et un conseil de surveillance composé de 6 membres, dont 2 issus des ministères et 4 de la sphère privée. Ce conseil a un devoir de contrôle interne des activités du conseil d'administration. Il participe à la prise de décisions importantes relatives au port et doit agir de manière indépendante, dans l'intérêt du port et de l'ensemble des actionnaires. Les superstructures et les équipements sont détenus et exploités par des entreprises privées. Enfin, à Tallinn, si la municipalité est absente des organes de direction, la coopération entre ville et port semble réelle et active. 
«Le port doit être le Gateway de Tallinn et de l'Estonie... la coopération est obligatoire, car si le port est un Gateway, quand vous entrez, vous devez avoir les directions, l'accès aux transports..." Maarika Liivamägi, membre du conseil de surveillance du port de Tallinn, octobre 2018. passagers au contact direct de la ville (figure 5) et les fonctions marchandes situées à Muuga, à une vingtaine de kilomètres de l'espace urbain, constitue surement un facteur explicatif de cette coopération.

Figure 5. Tallinn, terminal roulier et croisière en cœur de ville

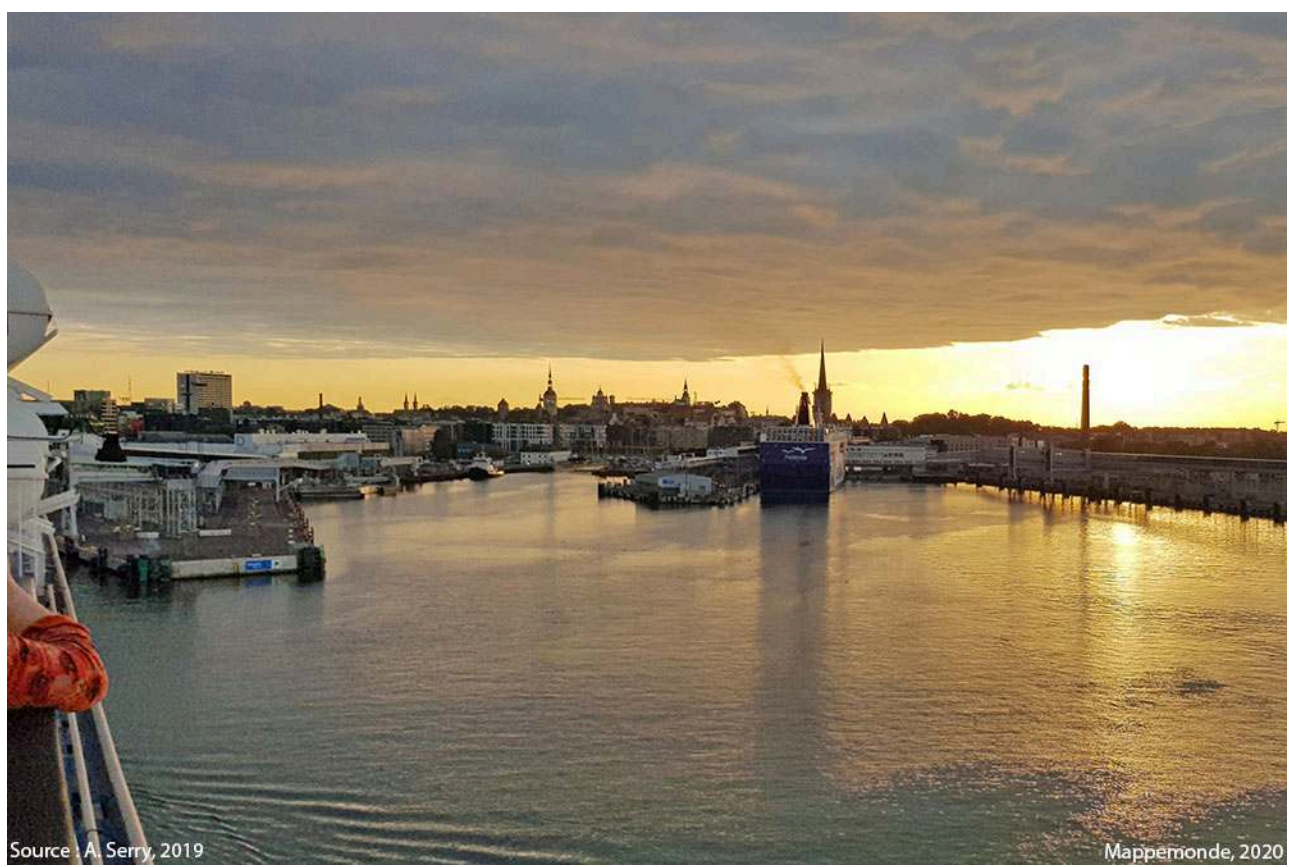

A. Serry

En Lituanie, la loi de 1996 définit les fonctions et l'organisation du port de Klaipeda. Sous tutelle directe du ministère des transports lituanien (figure 4), l'autorité portuaire a pour principales missions de gérer son territoire, assurer la sureté et la sécurité, construire les infrastructures et produire les plans de développement stratégique. Le directeur du port est nommé par le ministre des Transports.

Le conseil de développement portuaire détermine la stratégie de développement et coordonne les relations entre l'autorité portuaire, municipale et les institutions gouvernementales. Il est composé de représentants des ministères des Transports, des Finances, de la Région, de la municipalité de Klaipeda, des représentants du monde académique, du port et de ses utilisateurs. Le conseil portuaire, quant à lui, n'a pas de fonction de supervision. Il est constitué de représentants issus des mêmes corps que ceux observés dans le cas du conseil de développement portuaire.

La figure ci-dessous (figure 6) présente l'organisation décisionnelle et la structuration du leadership selon les représentations des acteurs portuaires interrogés. Elle résulte des entretiens conduits en 2017 auprès de différents acteurs de Klaipeda. Plus un acteur se positionne en haut du schéma et davantage il influence les décisions en matière d'orientations portuaires. 
Figure 6. Les acteurs de la gouvernance de la place portuaire de Klaipeda

Haut niveau de leadership

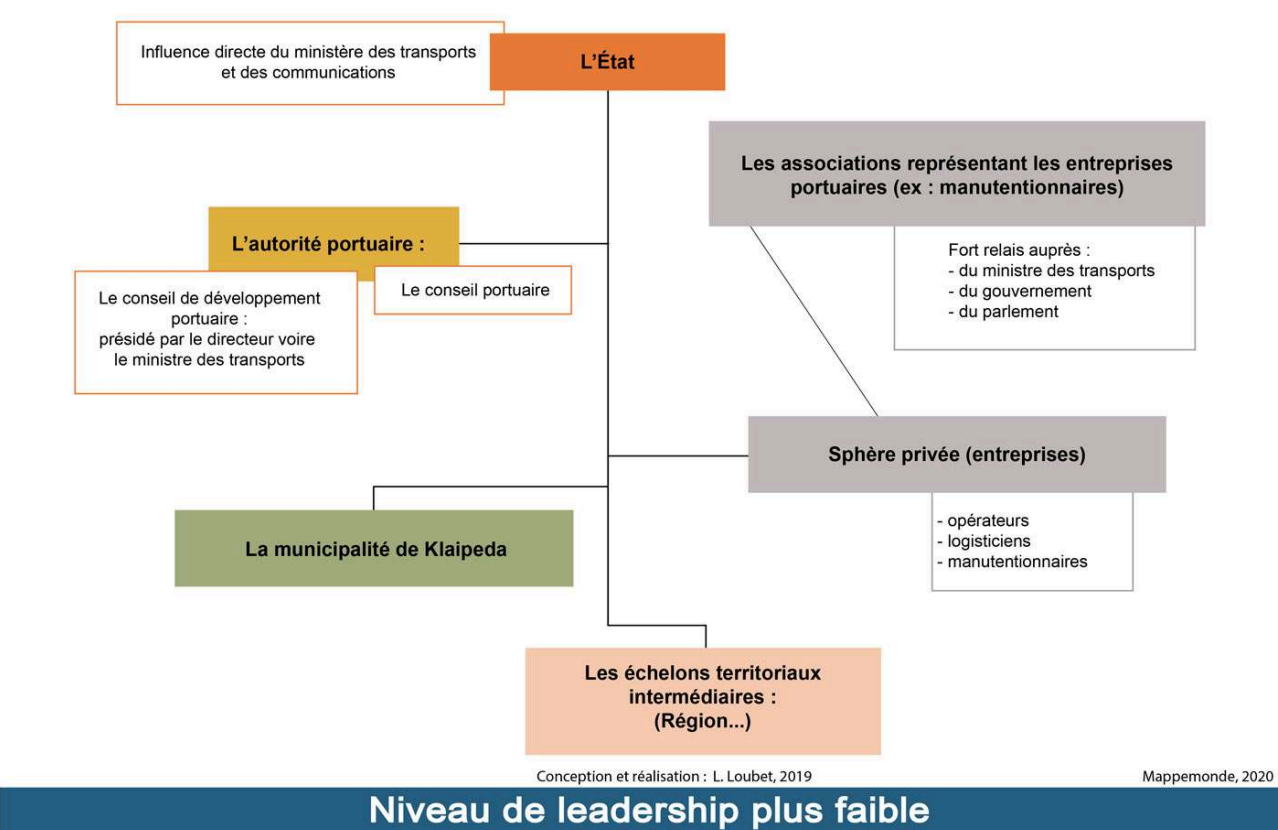

Réalisation : L. Loubet

Ainsi, la gestion institutionnelle du port de Klaipeda laisse apparaitre un État disposant

d'un leadership supérieur à ce que nous pouvons observer dans les autres États baltes.

«Nous sommes une entreprise d'État et avons été établis par le ministre des

Transports. Nous sommes comme une filiale du ministère des Transports ». Un

responsable de l'autorité portuaire de Klaipeda, avril 2017.

L'analyse du discours des acteurs confirme la prégnance du leadership de l'État (figure 7). En effet, les entretiens ont été intégrés dans deux logiciels d'analyse textuelle (NVIVO et ALCESTE), afin de construire un nuage des mots les plus utilisés sur l'ensemble des entretiens afin d'identifier ceux faisant référence aux liens ville-port dans un cadre territorial élargi ou de créer des réseaux de formes autour de trois mots clés : « ville », « port » et « gouvernance » afin d'identifier les mots les plus reliés avec eux dans le discours. 


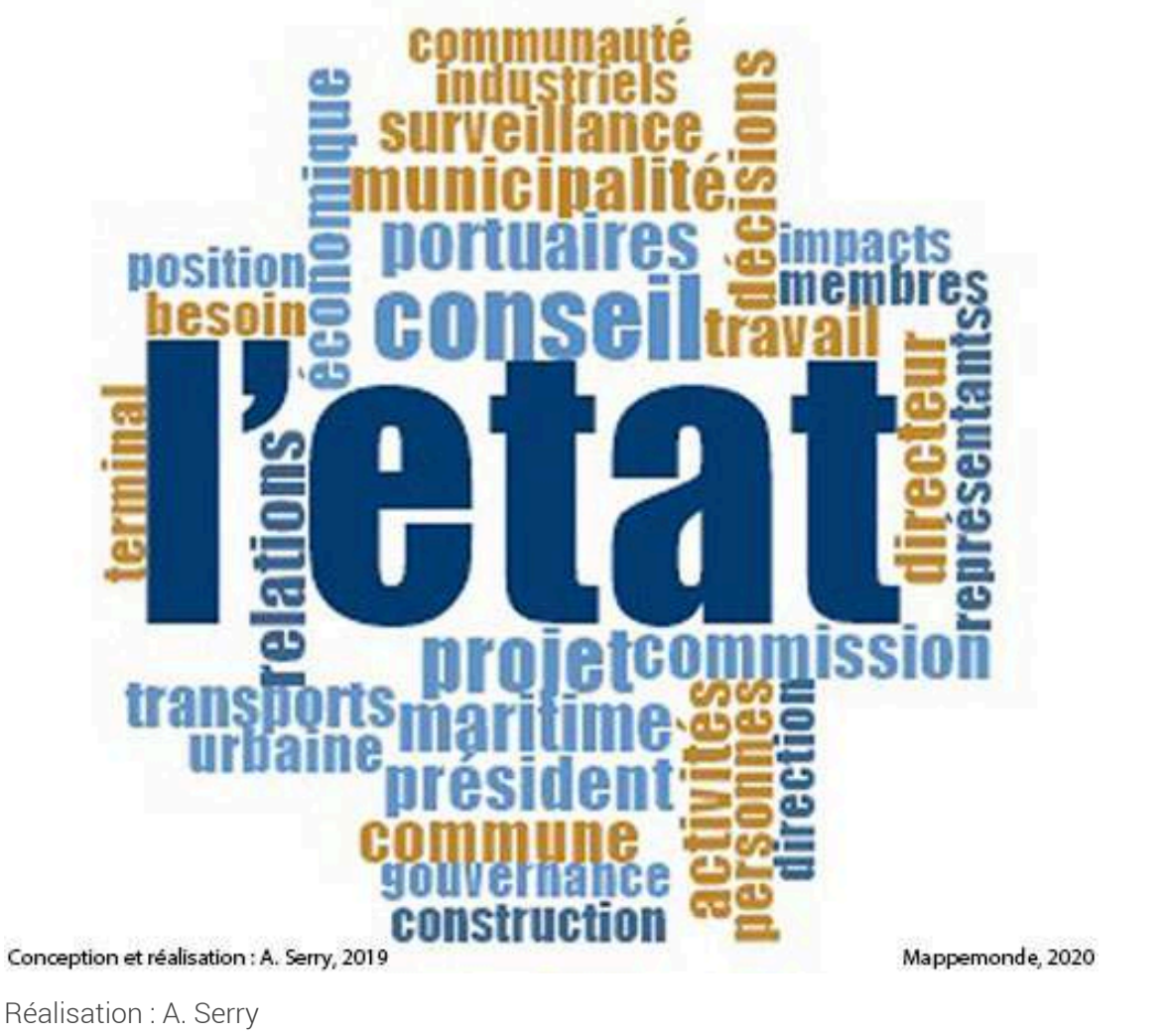

Le port de Klaipeda a mis en place un plan de réformes similaire à celui de Tallinn tout en valorisant une démarche de commercialisation plutôt que de corporatisation (figure 1). Aussi, l'État garde le contrôle de l'organisation portuaire, mais dans un environnement «pro-business » avec une certaine autonomie de management et de responsabilité (Bolevics et Volkoka, 2011). Ainsi, les acteurs de la sphère privée sont des partenaires importants présents dans la majorité des scènes décisionnelles. Au moyen d'associations de défense de leurs intérêts, les entreprises participent activement à la gestion du port :

«C'est notre but de développer le port de Klaipèda ensemble avec les institutions étatiques, l'autorité portuaire de Klaipèda...» Un directeur d'entreprise de manutention, avril 2017.

La municipalité quant à elle n'est pas reconnue par l'autorité portuaire comme un acteur portuaire :

« Il n'y a pas de problèmes [pour se mettre d'accord], mais des discussions avec la municipalité ; mais ce n'est pas un acteur portuaire ». Un responsable de l'autorité portuaire de Klaipeda, avril 2017.

Avec une population de 148900 habitants en 2018, Klaipeda est la troisième ville de Lituanie, une ville confrontée au phénomène de shrinking cities. Même si Klaipeda a vu sa population diminuer de 19,3\% entre 1993 et 2010, ce déclin urbain est particulièrement dû à la périurbanisation (Spiriajevas et Beteika, 2015). Par ailleurs, cette perte de population ne semble pas être synonyme de perte d'activité notamment parce qu'environ 800 entreprises comptant un effectif de plus de 23000 personnes sont engagées dans des activités liées au port, dont 10300 localisées dans la ville de Klaipeda. L'activité portuaire (46,3 Mt en 2018) y a connu une vive croissance de son 
trafic depuis l'indépendance du pays au début des années 1990, notamment parce que s'y développent des trafics diversifiés (vrac liquide, vrac sec comme des engrais, conteneurs, rouliers).

En ce qui concerne l'interface ville-port, à Klaipeda le port et la ville sont encore étroitement imbriqués (figure 8). L'activité portuaire est fortement contrainte dans son développement par la morphologie urbaine. D'un autre côté, l'accès à la mer pour la ville est contraint par le territoire portuaire. Fonction portuaire et fonction urbaine sont contiguës, n'offrant que peu de friches portuaires à des projets de requalification. Les impacts sociaux et environnementaux (émissions de poussières notamment) de l'activité portuaire y sont d'ailleurs très nets. Les projets de développement infrastructurels pour accompagner la croissance de trafic cristallisent aujourd'hui les tensions en plus des traditionnels conflits liés à la pollution par les poussières de charbon dans la partie nord du port (à proximité du centre-ville) ou des nuisances sonores le long des axes de pénétration vers les terminaux rouliers ou de conteneurs.

Figure 8. Ville et port à Klaipeda

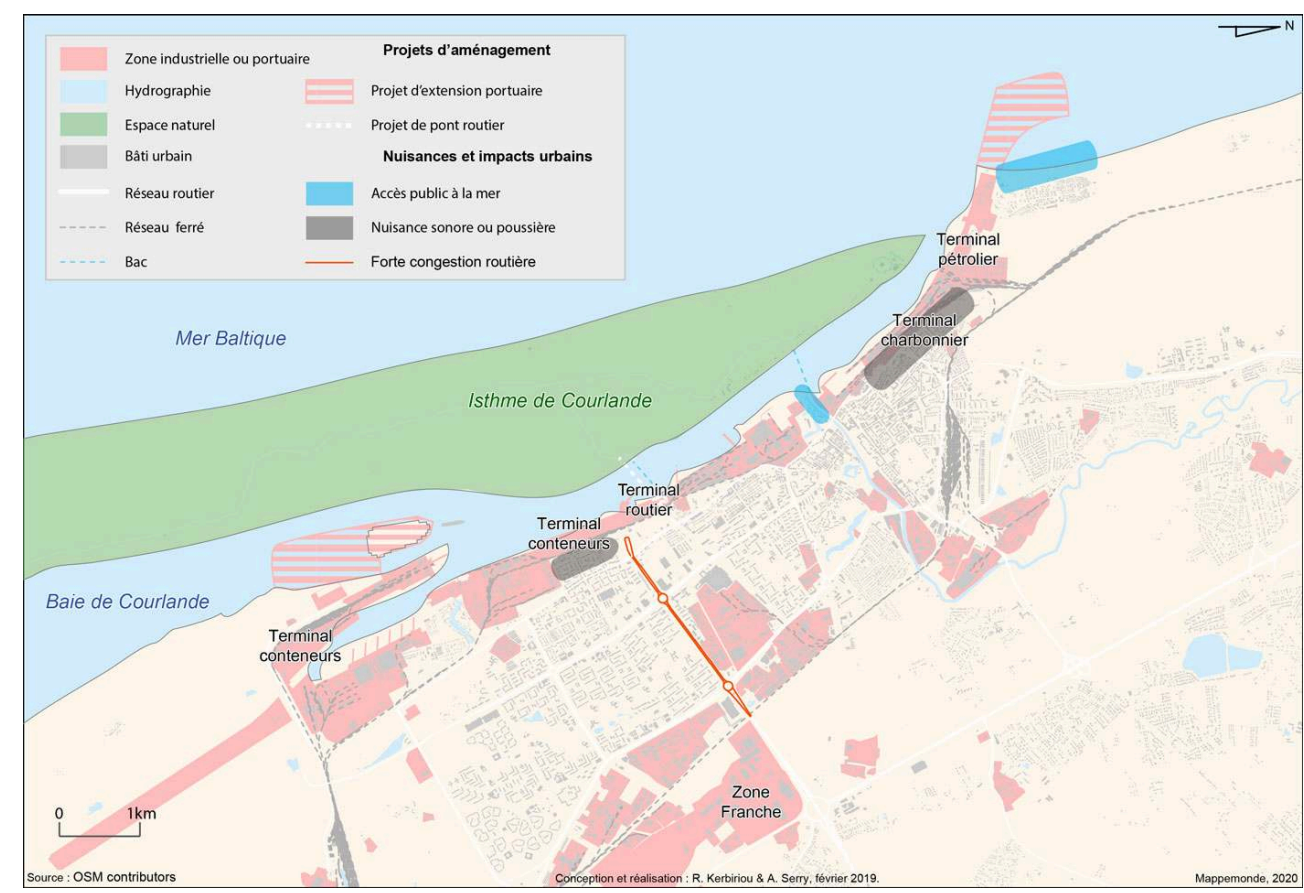

Réalisation : R. Kerbiriou et A. Serry

\section{La spécificité finlandaise}

Les installations de Kotka et Hamina sont les plus à l'est de la Finlande et font ainsi partie intégrante de cette rangée portuaire en proximité avec la Russie (figure 2). La configuration y est foncièrement différente des situations observées dans les États baltes.

Avant de fusionner en mai 2011, ces ports ont été les deux premiers de Finlande à être administrés par des sociétés municipales, entreprises commerciales sans obligations sociétales, contrairement aux entreprises municipales qui gèrent beaucoup de ports 
finlandais (figure 4). Elles ne reçoivent aucun soutien financier, peuvent faire des profits et offrir un rendement raisonnable à la municipalité (Finnila et al., 2011).

« Nous sommes propriété de la municipalité, bien sûr nous lui fournissons des revenus » Ville Kuitunen, directeur commercial du port d'Hamina-Kotka, décembre 2018.

Depuis la fusion, il s'agit d'une seule société anonyme, Port of HaminaKotka LTD, détenue à $60 \%$ par la municipalité de Kotka et $40 \%$ par celle d'Hamina. Elle administre les activités portuaires des deux villes. La société loue les entrepôts, les bureaux et l'espace au sol dans la zone portuaire.

«Le port est une société anonyme propriété de la ville, mais qui opère indépendamment. Il a son propre directeur, sa propre administration. La ville s'occupe de l'aménagement du territoire» Toni Vanhala, directeur $d u$ développement de la ville de Kotka, décembre 2018.

Le rôle de l'état finlandais dans la gouvernance du port de Kotka-Hamina est minime (figure 9). C'est aussi le seul port de notre étude à ne pas se définir uniquement comme un landlord port, mais plutôt comme une combinaison des trois types de ports, notamment en raison de son action dans les services portuaires et dans le financement de certains équipements.

Figure 9. Système de relations dans le port de Hamina kotka

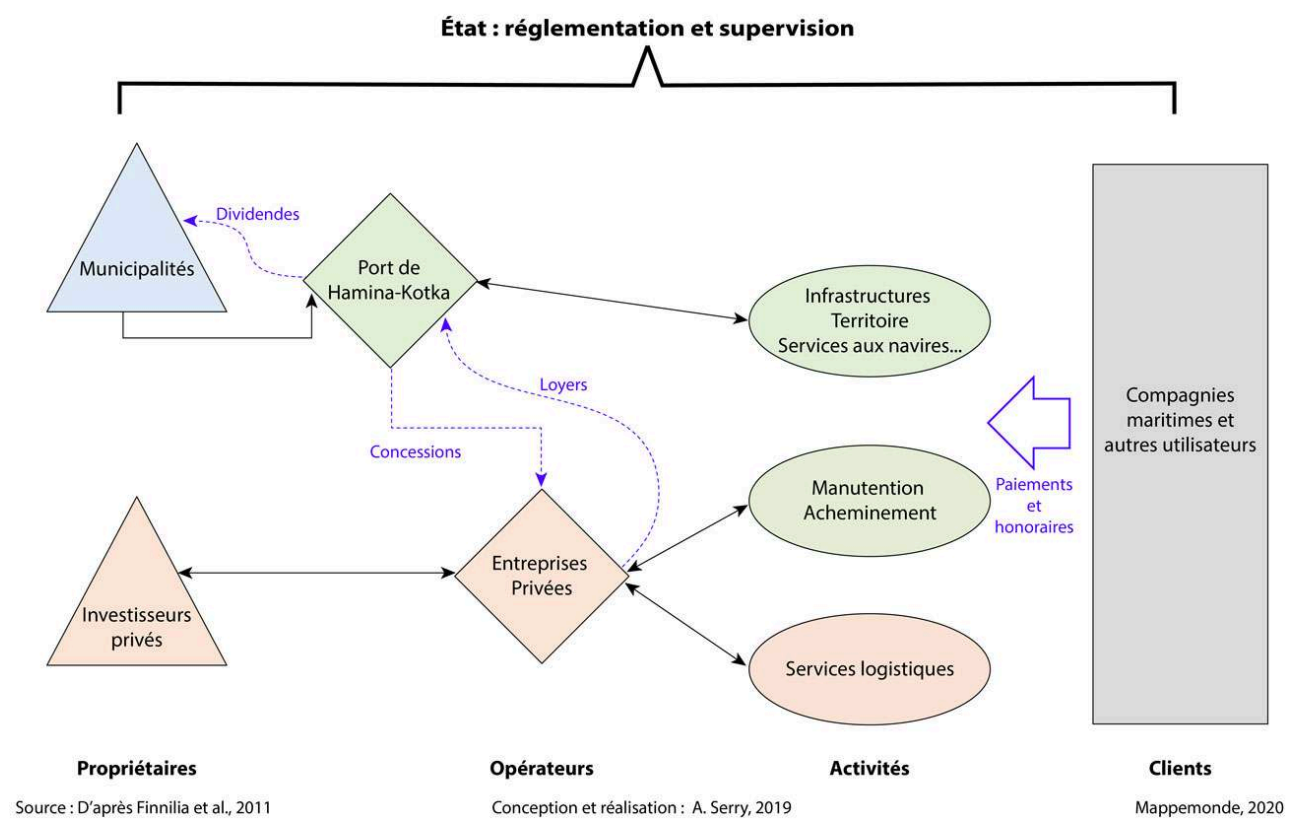

Réalisation : A. Serry d'après Finnila et al., 2011

\section{Les ports russes}

Dans un contexte chaotique, plusieurs années se sont écoulées avant que la Russie décide d'élaborer une politique maritime permettant une répartition plus lisible des compétences entre acteurs portuaires privés et publics (Pursiainen, 2007). L'État fédéral est le principal architecte de la politique portuaire russe. Cette omniprésence est souvent perçue et présentée comme un handicap, car synonyme de corruption. Le rôle des autorités locales dans le développement portuaire de la Russie est 
essentiellement limité à la mise en œuvre des décisions du gouvernement fédéral. Dans de nombreux cas, il existe un jeu de concurrence entre les sujets de la fédération : la région de Leningrad est en concurrence avec Saint-Pétersbourg.

Le secteur portuaire est en réalité largement détenu par les entreprises privées, en particulier celles positionnées sur l'exportation (pétrole, minerais...). La stratégie des grandes entreprises pétrolières est de construire leurs propres infrastructures afin d'assurer leurs besoins en transport et de minimiser les coûts d'exportation. De ce fait, après la privatisation, les sociétés commerciales des ports se sont retrouvées pour la plupart dans la sphère des géants de l'industrie russe. La compagnie pétrolière Lukoil est, par exemple, derrière le projet portuaire de Vyssotsk.

Dès 1994, un organisme public, dépendant directement du gouvernement russe, a été créé. Il est responsable de la gestion du port, de la navigation dans le port et dans la majeure partie du golfe de Finlande. Il est communément appelé «Grand port de SaintPétersbourg ». Sans activité commerciale, l'autorité portuaire est un organisme fédéral sans but lucratif qui exerce des fonctions régaliennes.

Si les ports de Primorsk et Oust-Louga sont dans le giron du "grand port de SaintPétersbourg " du point de vue de la navigation et de la sécurité, ils n'en restent pas moins aux mains d'investisseurs tiers. Primorsk est ainsi détenu et exploité par Transneft, entreprise publique russe qui dispose du monopole du transport de pétrole brut par conduites (Thorez, 2009). Vingt-huit entreprises de manutentions louent 200 quais dans ces ports (Anghel et Ciobanu, 2018). Elles ont en charge la manutention et les opérations d'accompagnement dans le port. La multiplicité des investisseurs regroupés sous la forme de la JSC Ust-Luga caractérise la situation à Oust-Louga. L'entreprise a en effet essayé d'avoir une situation quasi monopolistique en termes de services portuaires, voulant par ailleurs garder la propriété des terrains que les opérateurs souhaitaient acquérir.

Le port de Kaliningrad doit à sa localisation spécifique une évolution assez différente. Il est divisé entre quatre organismes principaux qui assurent des opérations en lien avec le commerce maritime : les ports de pêche, de commerce, fluvial et le port de Svelty. Si l'autorité portuaire assume le rôle qui lui est dévolu par l'état fédéral, les jeux d'acteurs $\mathrm{y}$ restent flous et la présence étrangère minime: en effet au-delà de la simple gouvernance portuaire et/ou urbano-portuaire, le rôle de l'État russe doit intégrer une dimension géopolitique qui se caractérise par le rôle de certaines compagnies privées ou la volonté affichée d'autonomie portuaire russe. Ainsi, les ambitions pour les ports russes, notamment Oust-Louga sont importantes, les investissements omniprésents ce qui en fait déjà le leader régional. Ces ports sont révélateurs de la mise en œuvre de la politique d'aménagement stratégique du littoral de la Baltique russe dans une logique de «nationalisation » des trafics entrants et sortants de la Russie, sans passer par les ports voisins étrangers (Serry, 2019). Par ailleurs, de nombreuses sociétés russes, tant locales que relayant des capitaux de Russie, prennent des parts importantes dans des entreprises baltiques. Par exemple, la compagnie russe Global Ports a investi plus de 500 millions d'euros en Estonie en 2014 via ses deux filiales Vopak E.O.S. AS et Global Trans Spacecom, principalement dans les terminaux pétroliers de Muuga. La compagnie est également présente dans les ports de Kotka et Helsinki. Cette omniprésence russe se manifeste également à une autre échelle illustrée par l'ouverture par Gefco, en collaboration avec la société russe Transcontainer, d'une nouvelle route multimodale 
connectant l'Allemagne et la Chine via Riga (le premier train constitué de 52 conteneurs de 40 EVP est parti de Riga le 8 mai 2017).

\section{Gouvernance portuaire et développement territorial}

51 Le développement territorial repose sur deux moteurs fondamentaux : la gouvernance et la production (Torre, 2018). Selon l'auteur, les relations entre acteurs sont au centre de ces deux dimensions. Au cœur de la gouvernance: matérialisant une mécanique décisionnelle territorialisée. Au cœur de «la production » structurée autour de deux dimensions centrales: l'innovation technologique et le caractère systémique des relations locales.

Dans le cas présent, les relations entre ports et territoires sont nombreuses et cet article constitue les prémices d'un programme de recherche-action ${ }^{2}$ qui vise à optimiser et conjuguer leur développement respectif. Au-delà d'une simple coexistence, l'enjeu réside dans la création de synergies bénéficiant aux deux entités. Le territoire doit former une ressource dans le développement économique portuaire. Réciproquement, le port doit participer au développement urbain/régional. Il ne s'agit plus de traiter deux processus distincts, qui entreraient en interaction de façon épisodique et indirecte, mais d'identifier les ressorts d'un enrichissement mutuel.

Dans le contexte régional, la gouvernance est un ressort primordial de la performance portuaire, devant le marché et le cadre légal (Bolevics, 2017). Elle est le produit de logiques actorielles, organisationnelles, mais également territoriales. En effet, bien que souvent le territoire appréhendé soit borné par une territorialité portuaire, il convient de considérer avec attention les dynamiques territoriales qui structurent le territoire élargi (commune d'accueil, région, État, grandes aires d'influence économiques et politiques). L'espace baltique ne fait pas exception. Il souscrit à un mouvement général de mutations du commerce international ayant transformé et éloigné les ports des villes. Cette prise de distance spatiale peut conduire à distendre les liens dans un contexte où "proximité spatiale » et "proximité cognitive » peuvent être corrélées (Bouba-Olga et Grossetti, 2008). Les acteurs municipaux, par exemple, évaluent plus difficilement les évolutions contemporaines de l'industrie maritime et la nécessité d'aménagements portuaires d'ampleur afin de demeurer compétitifs. Le découplage ville/port se renforce, alimenté par des logiques institutionnelles et organisationnelles propres à chaque acteur et territoire.

54 Ainsi, il n'existe pas de modèle type (Verhoeven, 2010). Toutefois, les ports de la Baltique orientale semblent pouvoir être catégorisés notamment au regard de la place accordée à l'échelon municipal :

- Les ports russes et lituaniens sont des ports d'État dans lesquels les municipalités sont quasiabsentes, si ce n'est à travers quelques tentatives de marketing territorial en Russie ou la présence dans des organes consultatifs en Lituanie.

- En Finlande, Estonie et Lettonie, l'autorité municipale est un acteur de la décision portuaire, à des niveaux certes différents : exclue du gouvernement en Estonie, mais en discussion permanente avec l'autorité portuaire, intégrée aux organes décisionnaires portuaires lettons et enfin actionnaire unique de l'entreprise portuaire en Finlande.

Dans ce contexte, l'articulation du projet urbain et du projet portuaire, la planification spatiale ou l'élaboration des documents d'urbanisme révèlent la nature des relations 
entre acteurs à mesure qu'ils représentent des enjeux fondamentaux de développement local.

« Notre conseil d'administration est constitué de personnes locales, ce sont des élus municipaux... donc ils comprennent ce que ça veut dire d'avoir un port pour les emplois, pour l'industrie... donc c'est facile pour nous... » Le directeur commercial du port d'Hamina-Kotka, décembre 2018.

Cette géopolitique de l'aménagement urbano-portuaire s'exprime assez classiquement par des conflits d'usages. À cette occasion, impératifs environnementaux, économiques, bien-être des habitants, pêche, activités logistiques et industrielles, etc., peuvent s'opposer au développement portuaire.

Dans le cas de Klaipeda, par exemple, le tourisme et la protection du cadre de vie, promus par la municipalité, rencontrent les externalités négatives et les besoins fonciers de l'activité portuaire. De plus, «l'emboitement» des projets semble pâtir d'une forme d'instrumentalisation de la question portuaire mobilisée par la mairie pour exprimer ses velléités de résistance face au pouvoir central. Le manque de reconnaissance réciproque aboutit à un développement qui gagnerait à davantage de concertation:

«Il y en a deux [projets]... car ils ne sont pas unis et il y a deux développements distincts, pas un seul». Le responsable de l'académie maritime de Lituanie, avril 2017.

Dès lors, projets urbains et projets portuaires vont occasionner des jeux et stratégies qui s'illustrent notamment au travers de tentatives d'appropriation d'espaces :

«La ville est toujours en train d'intimider le port pour récupérer l'accès à l'eau, pour augmenter sa surface sur les activités portuaires ». Un directeur d'entreprise lituanienne de manutention, responsable de LJKKA, avril 2017.

59 Cette gouvernance instable, où le leadership des pouvoirs locaux, centraux et portuaires est sans cesse débattu, renforce les zones d'incertitudes pour des acteurs économiques qui ont besoin de stabilité institutionnelle :

« Nous avons besoin d'une frontière légitime et claire pour le développement futur $\mathrm{du}$ port de Klaipeda. Le port et la municipalité ne veulent pas s'accorder... Nous voudrions bien fixer pour les 25 prochaines années les zones pour le développement futur ». Un autre directeur d'entreprise lituanienne de manutention, responsable de LJKKA, avril 2017.

Cependant si les projets (ici portuaires) génèrent de l'incertitude, ils véhiculent également des vertus cognitives. Ils incitent les élus, techniciens voire les habitants à questionner l'intégration du port dans le territoire et la manière dont celui-ci participe de leur construction identitaire. Ils constituent également un moyen d'éprouver l'habileté organisationnelle des collectivités dans un contexte de complexité accrue où il convient d'apprendre à coopérer (Loubet, 2012).

«Nous avons eu un projet il y a quelques années. Nous avons discuté avec le ministère de l'Environnement, mais aussi avec un représentant de la région, avec des personnes de la municipalité et du port d'Hamina-Kotka... si nous voulons conclure, toutes ces personnes différentes se sont assises autour de la même table et ont discuté de qui est responsable de quoi ». Olli-Pekka Brunilla, Chercheur à South-Eastern Finland University of Applied Sciences, décembre 2018.

61 Aussi, la reterritorialisation des ports oblige les villes à accompagner de nouvelles recompositions territoriales dans un contexte de coopération accrue : «La complexité et le caractère très contextuel des enjeux rendent indispensables la collaboration des 
différents acteurs et l'étude préliminaire des forces et faiblesses du territoire... La bonne entente ville-port apparait de ce fait indispensable » (Jugie, 2014).

Cependant là encore, échelles et contextes sont primordiaux. Un pays comptant plusieurs ports mènera une politique bien différente d'un autre, doté de peu de ports, ou de ports ayant une influence mineure dans l'économie nationale (Foulquier et Maugeri, 2014). À Tallinn par exemple, la réflexion sur la recomposition du port de la vieille ville et l'élaboration d'un plan directeur est révélatrice de ce besoin de coopération. Les représentants du port et le conseil municipal ont été associés dans des ateliers afin de définir une vision claire du futur front de mer.

«C'est une occasion unique pour nous de commencer à transformer la zone portuaire en plein cœur de Tallinn en une ville innovante et accueillante " Valdo Kalm, Directeur du Port de Tallinn, 31 janvier 2019 (https://www.espo.be/news/ port-pro-of-the-month-valdo-kalm-ee).

63 De même, les changements structuraux que provoque la logistique globale redéfinissent les relations entre le port et sa région (Comtois, 2014). Les ports prolongent désormais leurs activités et leurs implications fonctionnelles au-delà même de leurs frontières métropolitaines ou régionales (Prelorenzo, 2011) et dépendent d'un développement territorial dépassant l'espace et le secteur portuaire :

«J'ai toujours un objectif dans ma tête - atteindre un million de conteneur par an - et nous travaillons en ce sens. Bien sûr, avant d'y arriver beaucoup de conditions doivent être remplies dont une grande majorité n'est pas directement reliées au port lui-même » Ansis ZeltiN̦š, directeur du port de Riga, aout 2018 (http:// www.baltic-course.com/eng/direct_speech/?doc=142225).

64 Malgré cette interdépendance port-territoire, il persiste également une méconnaissance des bénéfices, particulièrement économiques, liés au trafic maritime sur les arrière-pays et l'ensemble du territoire.

Enfin, outre les relations entre pouvoir central et collectivités territoriales, il convient de considérer avec attention le rôle de la sphère privée dans les ports. Par exemple, si la politique portuaire russe vise clairement à une autonomie portuaire, nombreux sont les acteurs privés à effectuer d'autres choix. Beaucoup de sociétés russes ou biélorusses (Belaruskali), tant locales que relayant des capitaux de Russie, se développent dans les ports baltes. Ainsi, comme évoqué plus haut, la compagnie russe Global Ports a investi plus de 500 millions d'euros en Estonie en 2014 principalement dans les terminaux pétroliers de Muuga. La même année, Mine Zarechnaya a pour sa part investi 20 millions d'euros dans le terminal charbonnier (figure 10) de Ventspils (Pestich, 2016). Aussi, audelà des stratégies politiques, les comportements des acteurs privés symbolisent également la spécificité fonctionnelle de la façade portuaire balte. 


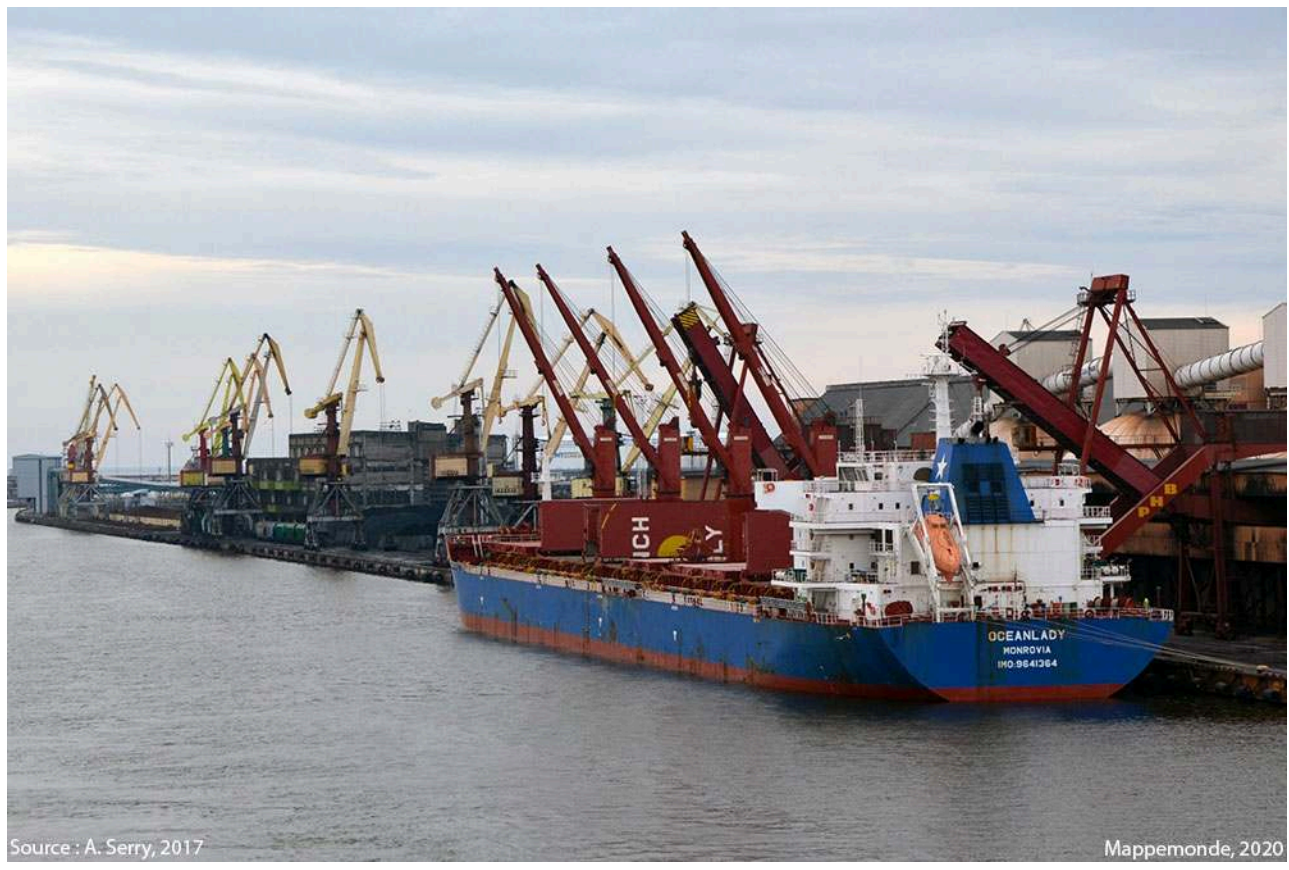

A. Serry

\section{Conclusion}

L'espace baltique sort d'une situation plutôt confortable de partage de la croissance. Celle-ci a favorisé sa revitalisation rapide. Mais aujourd'hui, dans un contexte de compétition accrue, les acteurs du monde maritime et portuaire doivent développer de nouvelles stratégies pour répondre aux enjeux régionaux contemporains.

Cependant, chaque port a son propre héritage culturel, historique et politique. De ce fait, il n'y a pas de gouvernance type. Les ports de Baltique orientale ont connu des réformes importantes depuis maintenant vingt ans. La tendance générale privilégie le modèle du landlord port dans lequel le partenariat public-privé est devenu primordial. Des différentiations ont vu cependant le jour dans les régulations portuaires. Une organisation proche du modèle hanséatique, fortement axée sur la « corporatisation ", se distingue en Estonie et en Finlande où la gestion des ports peut même être privée. En Russie et Lituanie, la gestion est assurée par une société publique, sous contrôle de l'État. En Lettonie, la situation est intermédiaire, laissant plus de place aux pouvoirs locaux. Toutefois, ces jeux, notamment ceux des entreprises privées restent flous.

Si l'analyse des cas baltiques ne suffit pas à opérer une montée en généralité, elle propose des pistes de réflexion qui pourraient permettre d'optimiser la gouvernance portuaire à des fins de développement territorial. Nos résultats semblent valoriser le rôle des acteurs, leur capacité à adopter des comportements coopératifs au sein de configurations territoriales spécifiques. Par exemple, il apparait que si la sphère entrepreneuriale participe amplement de la gouvernance territoriale et portuaire, son influence tend à varier considérablement selon les territoires.

Par ailleurs, les considérations géopolitiques sont nombreuses dans la région, notamment les relations avec la Russie. Alors que la communauté portuaire balte est 
ouverte aux investissements russes, les États et les opinions publiques y sont hostiles. Parallèlement, les investissements occidentaux en Russie sont le plus souvent masqués.

Il semble que le prochain défi du monde portuaire balte repose sur une réflexion en termes de communauté portuaire et ceci à diverses échelles: ville-port, rangée ou région portuaire. Dans ce cadre, la ligue hanséatique qui a profondément marqué le commerce entre pays riverains de la mer baltique, représente un exemple où la coopération entre des ports et des centres de commerce intérieur a produit des bénéfices tangibles tant pour le développement du commerce que pour les communautés locales et régionales.

\section{BIBLIOGRAPHIE}

ANGHEL A. R., CIOBANU C. (2018). Aspects regarding the evolution of the transport of containerized goods through the port of Constanta in context of European containerized trade. Actes de la conférence «SEACONF 2018 », Constanzae, 17-19 mai 2018.

BOLĒVICS V. (2017). “The impact of governance on the efficiency of the Baltic states' major ports". Journal of Business Management, $\mathrm{n}^{\circ}$ 14, p. 7-26.

BoLĒVICS V., VolKoVA T. (2011). “The changing landscape of port governance: case of the Baltic states". Journal of Business Management, $\mathrm{n}^{\circ}$ 4, p. 162-169.

BOUBA-OLGA O., GROSSETTI M. (2008). « Socio-économie de la proximité ». Revue d'Économie régionale et urbaine, $\mathrm{n}^{\circ}$ 3, p. 311-328.

BROOKS M.-R., PALLIS A.A. (2012). "Port Governance”. In TALLEY W.K., The Blackwell Companion to Maritime Economics, Hoboken : Wiley-Blackwell, p. 491-516. ISBN 978-1-444-33024-3

BURNS M.-G. (2014). Port management and operations. Boca Raton : CRC Press, 406 p.

CHARLIER J., LAVAUD-LETILLEUL V. (2011). « Zeebrugge ou l'émergence d'une nouvelle porte océane au cœur du Northern Range ». Territoire en mouvement, $\mathrm{n}^{\circ} 10$. En ligne : http://

journals.openedition.org/tem/1071

ComTois C. (2014). « Les échelles géographiques à la gouvernance ville-port ». In ALIX et al., Port City Governance. Caen : EMS, coll. « Les océanides », p. 39-54. ISBN 978-2-84769-684-4

FINNILA K., RONTY J., NOKKALA M. (2011). Port ownership and governance models in Finland. VTT Technical Research Centre of Finland, $105 \mathrm{p}$.

FOULQUIER E., MAUGERI S. (2014). « La notion de communauté portuaire. Le cas des pays d'Europe du Sud ». In FOULQUIER E. et LAMBERTS C., Gouverner les ports de commerce à l'heure libérale. Paris : CNRS Éditions, p. 17-36. ISBN 978-2-271-07654-0

FOULQUIER E. (2009). «Tensions, conflits et gouvernance dans les ports de commerce français (2004-2007) ». In DAMIEN M.-M et MARCADON J., coord., Les ports européens et la mondialisation. La réforme française, Paris : L'Harmattan, p. 97-118. 
GUERLET G. (2013). La gestion des ports par une entité publique : aspects européens et environnementaux. Université du Littoral Côte d'Opale, thèse de droit, 290 p.

GUILLAUME J. (2014). « L'État, entrepreneur portuaire ». In FOULQUIER E. et LAMBERTS C., Gouverner les ports de commerce à l'heure libérale. Paris : CNRS Éditions, p. 17-36. ISBN 978-2-271-07654-0

JUGIE J.-H. (2014). « L'approche urbaine de la gouvernance ville-port ». In ALIX et al., Port City Governance. Océanides, Caen : EMS, p. 23-38. ISBN 978-2-84769-684-4

KUKRUS A., POLDROOS J. (2003). "Infrastructure liberalization on the example of the Port of Tallinn". In Eesti majanduspoliitika teel Euroopa Liitu. Berlin : Verlag A. Spitz, p. 113-120.

LEMAIRE O. (2012). Le défi ville-port. Note de synthèse de l'ISEMAR, $\mathrm{n}^{\circ} 146$. En ligne : http:// www.isemar.fr/wp-content/uploads/2016/11/note-de-synthese-isemar-146.pdf

LÉVÊQUE L. (2014). « Du cluster portuaire au système portuaire régional : des territoires structurés par la gestion des flux de marchandises ». Actes du colloque «CIST2014 - Fronts et frontières des sciences du territoire ", Paris, 27-28 mars 2014.

LOUBET L. (2012). « L'apprentissage de la coopération intercommunale : modalités et instruments ». L'espace politique, vol. 18, $\mathrm{n}^{\circ}$ 3. En ligne : http://espacepolitique.revues.org/ index $2454 . h t m l$

MICKIENE R., VALIONIENE E. (2017). "Evaluation of the interaction between the state seaport governance model and port performance indicators". Forum Scientiae Oeconomia, vol. $5, \mathrm{n}^{\circ} 3$, p. 27-43.

PESTICH A.-S. (2016). "Russian's role in the Baltic States' investment". Journal of Geography, Politics and Society, vol. 6, $n^{\circ}$ 2, p. 68-75.

PRELORENZO C. (2011). « La ville portuaire, un nouveau regard ». Rives méditerranéennes, $\mathrm{n}^{\circ}$ 39, p. 13-22.

PURSIAINEN C. (2007). Russia between integration and protectionism: International road transport, ports, and the forestry sector. Nordregio working paper, Stockholm, $79 \mathrm{p}$.

RODRIGUE J.-P. et al. (2017). The Geography of Transport Systems. New York : Routledge, 440 p. ISBN 978-1138669574

SERRY A. (2018). Transport maritime en Baltique, de la complémentarité à la concurrence. Note de synthèse de l'ISEMAR ; n 196. En ligne : https://www.isemar.fr/wp-content/uploads/2018/01/ note-de-synthese-196-baltique.pdf

SERRY A. (2019). « Connecter l'espace baltique : enjeux et perspectives ». In SERRY A., ALIX Y. SENCILA V., Baltic-Arctic/Strategic perspective, Caen : EMS, p. 133-158. ISBN 978-2-37687-329-7

SLACK B. (2007). “The terminalisation of seaports”. In WANG J., OLIVIER D., NOTTEBOOM T. et SLACK B., Port, cities and global supply chains. Londres : Ashgate Publishing Limited, p. 41-50.

SPIRIAJEVAS E., BETEIKA L. (2015). "Spatial patterns of crimes in Klaipeda and their assessment in social geographic approach”. Criminological Studies, vol. 3, p. 124-153.

THOREZ P. (2009). «Le renouveau portuaire de la Russie en mer Baltique ». In DAMIEN M.-M. et MARCADON J., Les ports européens et la mondialisation, Paris : L'Harmattan, p. 83-94.

ISBN 978-2-296-09163-4

TORRE A. (2018). « Les moteurs du développement territorial ». Revue d'Économie régionale et urbaine, $\mathrm{n}^{\circ} 4$, p. 711-736. 
TOURRET P. (2014). « Le modèle hanséatique entre mythes et réalités ». In FOULQUIER E. et LAMBERTS C., Gouverner les ports de commerce à l'heure libérale, Paris : CNRS Éditions, p. 183-196.

VERHOEVEN P., VANOUTRIVE T. (2011). A quantitative analysis of European port governance. Conférence « Econship », Chios, 22-24 juin 2011.

VERHOEVEN P. (2010). European Port Governance. European Sea Ports Organisation. En ligne : https:// www.espo.be/media/espopublications/espofactfindingreport2010.pdf

\section{NOTES}

1. Le guide d'entretien utilisé pour cette enquête se compose de plusieurs sous-parties communes à tout type d'acteurs, à savoir le rôle et la place de l'acteur au sein de la place portuaire, les impacts de l'activité municipale/intercommunale sur l'organisation et les activités de l'acteur (ou, le cas échéant, les impacts de l'activité portuaire sur l'organisation et les activités de l'acteur), le processus décisionnel au sein de la gouvernance portuaire, la nature des forces en présence dans le cadre de l'organisation portuaire, les relations avec les autres territoires.

2. RIN recherche : «PORTERR - Ports et territoires », (resp. Serry et Loubet) financé par le Conseil Régional de Normandie ; partenaires : Université Le Havre-Normandie/UMR CNRS IDEES/INSA de Rouen/EMN/NEOMA.

\section{RÉSUMÉS}

Depuis une vingtaine d'années, la mer Baltique a été touchée par de multiples bouleversements d'abord géopolitiques, mais également économiques. Ceux-ci ont notamment obligé les ports à se transformer et à s'adapter aux évolutions de l'industrie maritime. Les ports de la Baltique orientale sont passés d'une situation de complémentarité, instruments du collectivisme soviétique, à une intégration dans le système maritime et portuaire mondial synonyme de libéralisme. Aussi, la question de la modification de la gouvernance et des régulations portuaires au sein d'une région en pleine mutation intéresse particulièrement l'espace baltique. Une revue de littérature ainsi qu'un travail de terrain appuyé sur une vingtaine d'entretiens (auprès d'élus, représentants des autorités portuaires, directeurs d'entreprises de manutention, responsables de chambres de commerce, lobbyistes...) permettent de mettre en lumière la manière dont les jeux d'acteurs structurent les places portuaires et impactent le développement des territoires (portuaires, urbains, arrière-pays).

Over the past two decades, the Baltic sea has known multiple upheaveals, both political and economic. They have notably forced ports to change and adapt to the evolving maritime industries. The ports of the Eastern Baltic have gone from a complementary position as instruments of Soviet collectivism to becoming integrated within a global maritime and portuary system that is synonymous with liberalism. In addition, the issue of modifying governance and portuary regulations, within a rapidly evolving area, is of particular interest to the Baltic space. A literature review and a fieldwork based on two dozen interviews (with elected officials, representatives of port authorities, directors of dock handling companies, heads of chambers of 
commerce, lobbyists...) highlights how the choices of actors structure ports and affect territorial development (ports, urban areas, inland).

Desde hace dos décadas el mar Báltico se ha visto afectado por múltiples conflictos geopoliticos y económicos, que han obligado a los puertos a transformarse y adaptarse a los cambios del sector marítimo. Los puertos del Báltico oriental han pasado de ser instrumentos complementarios del colectivismo soviético a su integración, en un contexto de liberalismo, como parte del sistema marítimo y portuario mundial. En una zona en profunda transformación es muy relevante la modificación de la normativa y el modelo de gobernanza portuario.

INDEX

Keywords : Baltic, governance, port, actors, territory

Mots-clés : Baltique, gouvernance, port, acteurs, territoire

Palabras claves : Báltico, gobernanza, pueto, actores, territorio

Thèmes : La Baltique. Un espace d'intégration mais aussi de concurrence et de rivalités

\section{AUTEURS}

ARNAUD SERRY

Maître de conférences en Géographie, Université Le Havre Normandie, CNRS, UMR IDEES

\section{LILIAN LOUBET}

Maître de conférences en Aménagement et Urbanisme, Université Le Havre Normandie, CNRS, UMR IDEES 